\title{
Pressões respiratórias máximas na predição de risco pós-operatório em cirurgia cordíaca
}

\author{
Maximal respiratory pressures in predicting postoperative risk in \\ cardiac surgery
}

\author{
Karilin Tereza Santiago de Oliveira'; Tania Fernandes Campos²; Raíssa de Oliveira Borja ${ }^{3}$; \\ Diana Amélia de Freitas ${ }^{4}$; Karla Morganna Pereira Pinto de Mendonça ${ }^{5}$ \\ ${ }^{1}$ Fisioterapeuta - UFRN. Natal, RN - Brasil. \\ ${ }^{2}$ Doutora em Psicobiologia - UFRN. Natal, RN, Professora Adjunta IV do Departamento de Fisioterapia - UFRN. Natal, RN - Brasil. \\ ${ }^{3}$ Mestre em Fisioterapia pelo Programa de Pós-Graduação em Fisioterapia - UFRN. \\ Natal, RN, Fisioterapeuta do Hospital Estadual Dr. Ruy Pereira dos Santos. Natal, RN - Brasil. \\ ${ }^{4}$ Mestranda pelo Programa de Pós-graduação em Fisioterapia - UFRN, Bolsista CAPES. Natal, RN - Brasil \\ Doutora em Ciências da Saúde - UFRN, Professora Adjunta IV do Departamento de Fisioterapia - UFRN. Natal, RN - Brasil \\ Endereço para correspondência \\ Karla Morganna Pereira Pinto de Mendonça \\ Av. Senador Salgado Filho, 3000, Lagoa Nova \\ 59078-970 - Natal - RN [Brasil] \\ kmorganna@ufrnet.br
}

\begin{abstract}
Resumo
Introdução: As complicações pulmonares no pós-operatório de cirurgias cardíacas podem estar relacionadas à fraqueza muscular respiratória. Objetivos: Avaliar se os valores para as pressões respiratórias máximas podem ser preditores de complicação pulmonar pós-operatória e/ou maior permanência hospitalar em pacientes submetidos à cirurgia cardíaca. Métodos: Quatorze pacientes submetidos à cirurgia cardíaca foram avaliados no pré-operatório e divididos em dois grupos, A (pacientes com pressões respiratórias máximas $\otimes 75 \%$ do predito) e B (pacientes com pressões respiratórias máximas $\geq$ a $75 \%$ do predito). Resultados: O grupo B apresentou um número menor de dias de internação hospitalar $(\mathrm{p}=0,03)$. Verificaram-se $20 \%$ de incidência de complicação pulmonar no grupo A. Conclusões: Não foi possível inferir, na amostra avaliada, que a força muscular respiratória foi preditora de complicação pós-operatória. Entretanto, os pacientes com fraqueza muscular respiratória, diagnosticada previamente à cirurgia cardíaca, tenderam a permanecer internados por um período de tempo mais prolongado.
\end{abstract}

Descritores: Cirurgia torácica; Circulação extracorpórea; Complicações pósoperatórias; Músculos respiratórios.

\footnotetext{
Abstract

Introduction: Pulmonary complications in postoperative of cardiac surgery may be related to respiratory muscle weakness. Objective: To assess whether the low values for maximal respiratory pressures can be predictors of postoperative pulmonary complication and or longer hospital stay in patients undergoing cardiac surgery. Methods: Fourteen patients undergoing cardiac surgery were evaluated in the preoperative and divided into two groups, A (patients with maximal respiratory pressures $<75 \%$ of the predicted values) and $B$ (patients with respiratory muscle strength $\geq 75 \%$ of the predicted values). Results: Group B showed a lower number of hospital stay $(p=0.03)$. The incidence of pulmonary complications was $20 \%$ in group A. Conclusion: It was not possible to conclude, in the population studied, whether respiratory muscle strength was predictor of postoperative complication. However, patients with respiratory muscle weakness diagnosed previously to heart surgery tended to stay in hospital for a longer period of time. Key words: Extracorporeal circulation; Postoperative complications; Respiratory muscles; Thoracic surgery.
} 


\section{Introdução}

A cirurgia cardíaca é um método invasivo em que se propõe corrigir ou atenuar os sintomas decorrentes do funcionamento inadequado do coração. Contudo, índices significativos de morbidade e mortalidade estão ligados às complicações pulmonares provenientes desse procedimento ${ }^{1,2}$.

As principais complicações pulmonares pós-operatórias encontradas são: pneumonia, traqueobronquite, atelectasia, insuficiência respiratória aguda, ventilação mecânica por mais de 48 horas e broncoespasmo ${ }^{3-6}$. Essas, em grande parte, decorrem de alterações pulmonares que podem ser agrupadas em quatro categorias: a) alterações na mecânica pulmonar; b) alterações no padrão respiratório; c) alterações na troca gasosa e d) alterações nos mecanismos de defesa pulmonar ${ }^{7,8}$. Associado a esses fatores tem-se o uso da circulação extracorpórea, a qual potencializa a lesão pulmonar', uma vez que induz uma reposta inflamatória, contribuindo para o surgimento de atelectasias, aumento do shunt, diminuição da complacência pulmonar e da troca gasosa ${ }^{10-12}$.

Nas cirurgias torácicas, também é esperada uma redução nos volumes e capacidades pulmonares, tendo como principal causa a disfunção diafragmática pós-operatória ${ }^{13,14}$, que ocorre entre duas e oito horas após a cirurgia, retornando aos valores pré-operatórios em sete a dez dias, aproximadamente. Essas alterações, que ocorrem em resposta ao procedimento cirúrgico, podem evoluir para complicações respiratórias quando alteram o curso inicialmente previsto para recuperação pós-operatória7. Muitas dessas complicações podem estar relacionadas com a fraqueza dos músculos respiratórios ${ }^{15}$. Dessa forma, medidas simples e objetivas de disfunção diafragmática podem ser avaliadas por meio das pressões respiratórias máximas (pressão inspiratória máxima e pressão expiratória máxima), as quais constituem um índice essencial para o diagnóstico de fraqueza muscular respiratória ${ }^{16}$.
Atualmente, é pequeno o volume de pesquisas que avaliam a disfunção muscular respiratória previamente ao ato cirúrgico e suas implicações respiratórias no pós-operatório. Portanto, neste estudo, tem-se como propósito avaliar se os valores para as pressões respiratórias máximas abaixo do previsto podem ser preditores de complicação pulmonar pós-operatória e/ou maior permanência hospitalar em pacientes submetidos à cirurgia cardíaca com circulação extracorpórea.

\section{Material e métodos}

Este estudo caracteriza-se como observacional analítico de caráter prospectivo, o qual foi submetido ao Comitê de Ética em Pesquisa (CEP) do Hospital Universitário Onofre Lopes (HUOL) obtendo parecer favorável de número 100/2007. O grupo amostral foi composto por 14 pacientes, 6 mulheres e 8 homens, acima de 18 anos, que foram submetidos à cirurgia cardíaca eletiva no Hospital Universitário Onofre Lopes e no Hospital e Maternidade Promater. Foram excluídos do estudo os indivíduos submetidos à cirurgia cardíaca não eletiva, à cirurgia cardíaca sem o uso da circulação extracorpórea, os menores de 18 anos, os voluntários com história de acidente vascular cerebral (AVC), em uso de imunossupressores 30 dias antes da cirurgia, presença de desordens neuromusculares diversas, cirurgia pulmonar anterior, doença pulmonar obstrutiva crônica, instabilidade cardiovascular e existência de aneurisma. Todos os participantes assinaram o termo de consentimento livre e esclarecido (TCLE).

Os pacientes foram avaliados no pré-operatório e acompanhados no pós-operatório até a alta hospitalar por um único pesquisador. A rotina do atendimento fisioterapêutico não foi alterada.

\section{Avaliação pré-operatória}

Foi utilizada uma ficha contendo dados referentes à identificação pessoal, história clíni- 
ca e exame físico, tais como gênero, idade, tabagismo, sintomas respiratórios (tosse, dispneia e cansaço), doença pulmonar prévia, comorbidades (hipertensão arterial e diabetes melito), índice de massa corpórea (foi classificado como eutrófico o paciente com índice de massa corpórea entre 21 e 29; e distrófico, aquele com o índice igual ou abaixo de 20 ou acima de 29$)^{17}$ e a força muscular respiratória mensurada por meio das pressões inspiratórias e expiratórias máximas (PImáx e PEmáx, respectivamente).

Para mensurar as pressões respiratórias máximas, foi utilizado um manovacuômetro analógico da marca Ger $\mathrm{Ar}{ }^{\circledR}$, calibrado em $\mathrm{cmH}_{2} \mathrm{O}$, com limite operacional variando de $-300 \mathrm{a}+300$ $\mathrm{cmH}_{2} \mathrm{O}$. A avaliação foi realizada com paciente na posição sentada, utilizando um clipe nasal para oclusão das narinas. A pressão inspiratória máxima foi realizada a partir do volume residual, e a pressão expiratória máxima a partir da capacidade pulmonar total. As pressões medidas foram sustentadas por pelo menos um segundo. Foi realizada uma pequena abertura na extremidade distal do bocal (do tipo tubo de extremidade achatada com $2 \mathrm{~cm}$ de diâmetro interno, com orifício de $1 \mathrm{~mm}$ de diâmetro na extremidade superior), a fim de prevenir que os músculos faciais produzissem significantes pressões ${ }^{18}$. Além disso, durante a realização da pressão expiratória máxima, fez-se a contenção das bochechas manualmente pelo avaliador no intuito de minimizar o uso da musculatura acessória da face. Foram realizadas três manobras de pressão inspiratória máxima; e três de pressão expiratória máxima, considerando o maior valor das três medições ${ }^{19}$. Por ser um teste esforço-dependente foi fornecido encorajamento verbal ${ }^{20}$.

Os pacientes foram classificados em dois grupos, A e B, de acordo com os valores obtidos para a manovacuometria, os quais foram comparados com a tabela de normalidade proposta por Neder et al. ${ }^{21}$ Compuseram o grupo A cinco sujeitos que apresentavam PImáx ou PEmáx menor que $75 \%$ do previsto; e participaram do B nove pacientes que possuíam PImáx ou PEmáx maior ou igual a $75 \%$ do previsto ${ }^{22,23}$.

\section{Acompanhamento pós-operatório}

Durante o pós-operatório, o pesquisador realizou o acompanhamento dos pacientes diariamente até a alta hospitalar a fim de documentar a ocorrência das seguintes complicações pulmonares pós-operatórias: pneumonia (temperatura acima de $38^{\circ} \mathrm{C}$, sinais radiológicos de consolidação pulmonar e tosse produtiva); atelectasia com repercussão clínica evidente; broncoespasmo; ventilação mecânica prolongada, por mais de 48 horas ou necessidade de reintubação e assistência ventilatória mecânica; derrame pleural ou pneumotórax; e óbito decorrente de causa pulmonar ou não. O tempo de circulação extracorpórea foi coletado a partir da ficha do perfusionista.

\section{Anólise dos dados}

Os dados foram analisados por meio do programa SPSS 15.0 (Statistical Package for the Social Science), atribuindo-se o nível de significância de 5\%. A normalidade dos dados foi avaliada pelo teste Kolmogorov-Smirnov. Para avaliar diferenças entre os grupos A e B quanto aos valores obtidos para PImáx e PEmáx foi aplicado o teste " $\mathrm{t}$ " de Student não pareado e quanto às variáveis categóricas (idade, peso, altura, índice de massa corpórea e tabagismo) foi utilizado o teste de Fisher (usando a frequência). Com relação ao tempo de permanência hospitalar, foi utilizado o teste de Mann-Whitney (por meio da mediana) para comparar a diferença entre os grupos. As demais variáveis foram descritas por média e desvio-padrão.

\section{Resultodos}

A amostra foi composta por 14 sujeitos, sendo observada a predominância do sexo masculino (57\%) e média de idade de $55 \pm 16$ anos entre os indivíduos estudados. O peso médio foi $65 \pm 10 \mathrm{~kg}$, e a altura, 1,6 $\pm 0,1 \mathrm{~m}$ nos pacientes analisados. Com relação ao índice de massa corpórea, a média encontrada 
foi $26 \pm 3 \mathrm{~kg} / \mathrm{m}^{2}$ na amostra estudada. Do total dos participantes, $71 \%$ foram classificados como eutróficos; e 29\%, como distróficos. Os pacientes que apresentaram o índice de massa corpórea fora da faixa de normalidade pertenciam ao grupo B. Quanto ao tipo de cirurgia cardíaca, $43 \%$ dos pacientes realizaram cirurgia de revascularização do miocárdio; $43 \%$, de válvula; $7 \%$, de revascularização e válvula e $7 \%$ de comunicação interventricular. As cirurgias em sua totalidade foram realizadas com circulação extracorpórea, com duração média de $78 \pm 48$ minutos.

A análise comparativa entre os grupos A e $B$, com relação às variáveis categóricas (idade, peso, altura, índice de massa corpórea e tabagismo) não apresentou diferença estatisticamente significativa entre os grupos (Tabela 1).

Tabela 1: Comparação entre os grupos $A$ e $B$, quanto òs variáveis categóricas, verificada por meio do teste de Fisher

\begin{tabular}{ccccc}
\hline \multirow{2}{*}{ Variável } & \multicolumn{5}{c}{$\begin{array}{c}\text { Grupo A } \\
\mathbf{n}(\%)\end{array}$} & $\begin{array}{c}\text { Grupo B } \\
\mathbf{n}(\%)\end{array}$ & p valor \\
\hline \multirow{2}{*}{ Idade } & $>55$ anos & $3(60,0)$ & $4(44,0)$ & 0,500 \\
& $\leq 55$ anos & $2(40,0)$ & $5(55,6)$ & \\
\multirow{2}{*}{ Peso } & $>66,4 \mathrm{~kg}$ & $1(20,0)$ & $6(66,7)$ & 0,133 \\
& $\leq 66,4 \mathrm{~kg}$ & $4(80,0)$ & $3(33,3)$ & \\
\multirow{2}{*}{ Altura } & $>1,6 \mathrm{~cm}$ & $2(40,0)$ & $4(44,0)$ & 0,657 \\
& $\leq 1,6 \mathrm{~cm}$ & $3(60,0)$ & $5(55,6)$ & \\
IMC & $>26,1$ & $2(40,0)$ & $5(55,6)$ & 0,500 \\
& $\leq 26,1$ & $3(60,0)$ & $4(44,0)$ & \\
\multirow{2}{*}{ Tabagismo } & Sim & $2(40,0)$ & $3(33,3)$ & 0,622 \\
& Não & $3(60,0)$ & $6(66,7)$ & \\
\hline
\end{tabular}

IMC: índice de massa corporal.

A média do tempo de internação hospitalar em dias nos grupos A e B foi $10 \pm 8$ e $5 \pm 0,8$, respectivamente. Observou-se diferença significativa entre os grupos no número total de dias de internação $(p=0,03)$.

A média da PImáx no grupo A foi $58 \pm 25$ $\mathrm{cmH}_{2} \mathrm{O}$; e no $\mathrm{B}, 152 \pm 46 \mathrm{cmH}_{2} \mathrm{O}$. Com relação à PEmáx, observou-se a média de $108 \pm 74 \mathrm{cmH}_{2} \mathrm{O}$ no grupo A; e no B, $230 \pm 54 \mathrm{cmH}_{2} \mathrm{O}$. Houve diferença estatisticamente significativa, tanto para
PImáx ( $\mathrm{p}=0,001)$ como PEmáx $(\mathrm{p}=0,004)$, entre os grupos estudados.

Apenas um único paciente $(20 \%$ do grupo A) evoluiu com complicação pulmonar. Não foi relatado nenhum óbito e/ou perdas amostrais.

\section{Discussão}

A inexistência de diferença significativa entre os grupos estudados em relação às variáveis idade, índice de massa corpórea e tabagismo indica que os indivíduos de ambos os grupos, A e B, são semelhantes no tocante a esses fatores de risco, já consagrados na literatura científica. No entanto, observou-se que o tempo de circulação extracorpórea pode ter sido um fator de risco determinante para o aparecimento de complicação respiratória, uma vez que a paciente que apresentou tal complicação tinha o maior tempo de perfusão entre os indivíduos que compuseram o seu grupo. Esse achado está de acordo com o estudo de Beluda e Bernasconi ${ }^{24}$ que verificaram a associação entre a presença de complicações pulmonares e um maior tempo de circulação extracorpórea.

Os valores de referência propostos por Neder et al. ${ }^{21}$ para a população brasileira, quando adotados como critério para subdivisão dos pacientes estudados nos dois grupos descritos, demonstram pelas medidas das pressões respiratórias máximas uma diferença significativa entre os grupos, o que pode prover informações importantes na predição de risco de complicação pós-operatória na cirurgia cardíaca. Resultados semelhantes foram encontrados recentemente por Bellinetti e Thomson ${ }^{17}$, quando eles utilizaram os valores das pressões respiratórias máximas na classificação dos pacientes submetidos à cirurgia torácica e abdominal em expostos e não expostos. Esses autores afirmaram que os valores de PImáx podem ser preditores de complicações pulmonares por estarem associados à disfunção muscular pré-operatória. Em contradição, Hulzebos et al. ${ }^{25}$ não consideraram a PImáx no modelo que propuseram para escala 
de risco de complicações pulmonares em pacientes submetidos à revascularização do miocárdio. Esses pesquisadores incluíram PEmáx $\geq$ $75 \%$ como fator de proteção para complicações pós-operatórias.

De acordo com Hsia et al. ${ }^{26}$, pacientes que apresentam risco aumentado para desenvolver complicações pós-operatórias são aqueles que no pré-operatório possuem fraqueza muscular respiratória. Em concordância com este estudo, os achados aqui apresentados demonstram evidência de fraqueza muscular respiratória, avaliada previamente à intervenção cirúrgica, naquele paciente que apresentou diagnóstico conclusivo de complicação pulmonar pós-operatória.

Os resultados deste estudo também estão suportados por Nomori et al..$^{27}$, que observaram, ao utilizarem o valor absoluto de $80 \mathrm{cmH}_{2} \mathrm{O}$ como referência para as pressões respiratórias máximas em pacientes submetidos à cirurgia torácica, que os indivíduos cujas referidas pressões estavam abaixo desse valor tiveram complicações pulmonares pós-operatórias. Entretanto, esses autores não consideram as variáveis demográficas, que foram usadas neste estudo, tais como sexo e idade, para determinação dos valores previstos dessas pressões.

Os achados evidenciam ainda, quando se considera isoladamente o tempo de internação hospitalar entre os grupos estudados, que os indivíduos que apresentaram disfunção muscular respiratória no pré-operatório tenderam a um maior tempo de permanência hospitalar. Dados semelhantes foram encontrados no estudo de Bellinetti e Thomson ${ }^{17}$. Outros autores também observaram tais resultados ao estudarem uma amostra de pacientes com alto risco de complicações pulmonares pós-operatórias, que aqueles com maior força muscular respiratória, alcançada após treinamento muscular inspiratório préoperatório, apresentaram uma redução média de dois dias na permanência hospitalar ${ }^{28}$.

A inexistência de uma rotina de avaliação e intervenção fisioterapêutica pré-operatória, nos hospitais onde os dados foram coletados, foi con- siderada limitação para este estudo visto que influenciou diretamente no seu tamanho amostral.

A avaliação da força muscular respiratória previamente à cirurgia cardíaca pode prover informação importante e adicional acerca do risco cirúrgico ao qual o paciente encontra-se exposto, sobretudo pela facilidade e baixo custo característicos dessa avaliação. É importante enfatizar que a identificação pré-operatória de risco aumentado, em detrimento da disfunção muscular respiratória, pode fornecer informações úteis para o melhor preparo do paciente eletivo à cirurgia cardíaca. Estudos que avaliem, em um número maior de participantes, a associação entre as pressões respiratórias máximas pré e pós-operatórias, incluindo a interação dessas com outras variáveis (intensidade da dor, volumes e capacidades pulmonares e estado nutricional), poderão facilitar a identificação dos aspectos relativos à mecânica respiratória capazes de predizer risco de complicações pósoperatórias em cirurgia cardíaca.

\section{Conclusões}

Os resultados deste estudo não permitiram inferir que, na amostra avaliada, a força muscular respiratória foi preditora de complicação pós-operatória em cirurgia cardíaca. No entanto, estes achados foram sugestivos de que os pacientes com fraqueza muscular respiratória, diagnosticada previamente à cirurgia cardíaca, tenderam a permanecer internados por um período de tempo mais prolongado.

\section{Referências}

1. Arcêncio L, Souza MD, Bortolin BS, Fernandes ACM, Rodrigues AJ, Evora PRB. Cuidados pré e pós-operatórios em cirurgia cardiotorácica: uma abordagem fisioterapêutica. Rev Bras Cir Cardiovasc. 2008;23(3):400-10.

2. Williams-Russo P, Charlson ME, Mackenzie CR, Gold JP, Shires GT. Predicting postoperative pulmonary complications. Is it a real problem? Arch Intern Med. 1992;152(6):1209-13. 
3. Smetana GW. Preoperative pulmonary evaluation. N Engl J Med. 1999;340(12):937-44.

4. Filardo FA, Faresin SM, Fernandes ALG. Validade de um índice prognóstico para ocorrência de complicações pulmonares no pós-operatório de cirurgia abdominal alta. Rev Ass Med Brasil. 2002;48(3):209-16.

5. Hulzebos EH, Helders PJ, Favié NJ, De Bie RA, Brutel de la Riviere A, Van Meeteren NL. Preoperative intensive inspiratory muscle training to prevent postoperative pulmonary complications in high-risk patients undergoing CABG surgery: a randomized clinical trial. JAMA. 2006;296(15):1851-7.

6. Silva DR, Gazzana MB, Knorst MM. Valor dos achados clínicos e da avaliação funcional pulmonar pré-operatórios como preditores das complicações pulmonares pós-operatórias. Rev Assoc Med Bras. 2010;56(5):551-7.

7. Tisi GM. Preoperative evaluation of pulmonary function. Validity, indications, and benefits. Am Rev Respir Dis. 1979;119(2):293-310.

8. Saad IAB, Zambon L. Variáveis clínicas de risco préoperatório. Rev Ass Med Brasil. 2001;47(2):117-24.

9. Taggart DP, el-Fiky M, Carter R, Bowman A, Wheatley DJ. Respiratory dysfunction after uncomplicated cardiopulmonary bypass. Ann Thorac Surg. 1993;56(5):1123-8.

10. Conti VR. Pulmonary injury after cardiopulmonary bypass. Chest. 2001;119(1):2-4.

11. Wynne R, Botti M. Postoperative pulmonary and implications for practice. Am J Crit Care. 2004;13(5):384-93.

12. Andrejaitiene J, Sirvinskas E, Bolys R. The influence of cardiopulmonary bypass on respiratory dysfunction in early postoperative period. Medicina (Kaunas). 2004;40(sp. Issue):7-12.

13. Chinali C, Busatto HB, Mortari DM, Rockenbach CWF, Leguisamo CP. Inspirometria de incentivo orientada a fluxo e padrões ventilatórios em pacientes submetidos à cirurgia abdominal alta. Conscientiae Saúde. 2009;8(2):203-10.

14. Siafakas NM, Mistrouska I, Bouros D, Georgopoulos D. Surgery and the respiratory muscles. Thorax. 1999;54(5):458-65.

15. Elias DG, Costa D, Oishi J, Pires VA, Silva MAM. Efeitos do treinamento muscular respiratório no pré e pós-operatório de cirurgia cardíaca. Rev Bras Ter Intensiva. 2000;12(1):9-18.

16. McConnell AK, Copestake AJ. Maximum static respiratory pressures in healthy elderly men and women: issues of reproducibility and interpretation. Respiration. 1999;66(3):251-8.
17. Bellinetti LM, Thomson JC. Avaliação muscular respiratória nas toracotomias e laparotomias superiores eletivas. J Bras Pneumol. 2006;32(2):99-105.

18. Black LF, Hyatt RE. Maximal respiratory pressures: normal values and relationship to age and sex. Am Rev Respir Dis. 1969;99(5):696-702.

19. Brunetto AF, Alves LA. Comparing peak and sustained values of maximal respiratory pressures in healthy subjects and chronic pulmonary disease patients. J Pneumol. 2003;29(4):208-12.

20. Fiore Junior JF, Paisani DM, Franceschini J, Chiavegato LD, Faresin SM. Pressões respiratórias máximas e capacidade vital: comparação entre avaliações através de bocal e de máscara facial. J Bras Pneumol. 2004;30(6):515-20.

21. Neder JA, Andreoni S, Lerarioet MC, Nery LE. Reference values for lung function tests. II. Maximal respiratory pressures and voluntary ventilation. Braz J Med Biol Res. 1999;32(6):719-27.

22. Clanton TL, Diaz PT. Clinical assessment of the respiratory muscles. Phys Ther. 1995;75(11):983-95.

23. American Thoracic Society/ European Respiratory Society. ATS/ERS statement on respiratory muscle testing. Am J Respir Crit Care Med. 2002;166(4):518-624.

24. Beluda FA, Bernasconi R. Relação entre força muscular respiratória e circulação extracorpórea com complicações pulmonares no pós-peratório de cirurgia cardíaca. Rev Soc Cadiol Estado de São Paulo. 2004;14(sp. Issue):1-9.

25. Hulzebos EHJ, Meeteren NLUV, De Bie RA, Dagnelie PC, Helders PJM. Prediction of postoperative pulmonary complications on the basis of preoperative risk factors in patients who had undergone coronary artery bypass graft surgery. Phys Ther. 2003;83(1):8-16.

26. Hsia CC, Peshock RM, Estrera AS, Mcintire DD, Ramanathan M. Respiratory muscle limitation in patients after pneumonectomy. Am Rev Respir Dis. 1993;147(3):744-52.

27. Nomori HMD, Kobayashi RMD, Fuyuno GMD, Morinaga SMD, Yashima HRPT. Preoperative respiratory muscle training. Assessment in thoracic surgery patients with special reference to postoperative pulmonary complications. Chest. 1994;105(6):1782-8.

28. Hulzebos EHJ, Meeteren NLU, Buijs BJWM, Bie RA, Rivière $A B$, Helders PJM. Feasibility of preoperative inspiratory muscle training in patients undergoing coronary artery bypass surgery with a high risk of postoperative pulmonary complications: a randomized controlled pilot study. Clin Rehabil. 2006;20(11):949-59. 